\title{
Spatial Information Enhances Myoelectric Control Performance with Only Two Channels
}

\author{
Jiayuan He, Xinjun Sheng, Member, IEEE, Xiangyang Zhu, Member, IEEE, Chaozhe Jiang*, and Ning \\ Jiang*, Senior Member, IEEE
}

\begin{abstract}
Automatic gesture recognition (AGR) is investigated as an effortless human-machine interaction method, potentially applied in many industrial sectors. When using surface electromyogram (sEMG) for AGR, i.e. myoelectric control, a minimum of four EMG channels are required. However, in practical applications, fewer number of electrodes is always preferred, particularly for mobile and wearable applications. No published research focused on how to improve the performance of a myoelectric system with only two sEMG channels. In this study, we presented a systematic investigation to fill this gap. Specifically, we demonstrated that through spatial filtering and electrode position optimization, the myoelectric control performance was significantly improved $(p<0.05)$ and similar to that with four electrodes. Further, we found a significant correlation between offline and online performance metrics in the two-channel system, indicating that offline performance was transferable to online performance, highly relevant for algorithm development for sEMG-based AGR applications.
\end{abstract}

Index Terms-Automatic gesture recognition, myoelectric control, pattern recognition, electromyogram (EMG)

\section{INTRODUCTION}

A UTOMATIC gesture recognition (AGR) provides humans a convenient, natural way to interact with electronics and industrial equipment. Compared to the traditional button-based method (physical or virtual), AGR is simple and effortless, easy to be integrated into mobile and wearable devices. Recent studies demonstrated that AGR could be paired with the emerging virtual or augmented reality technology to enhance user experiences [1]. Currently, AGR is mainly applied in the field of rehabilitation engineering, such as assisting stroke patients with powered exoskeleton control [2], facilitating

This work was supported by the Natural Sciences and Engineering Research Council of Canada (Discovery Grant 072169); National Natural Science Foundation of China (Grant 51620105002 and 91748119), and State Key Lab of Railway Control and Safety Open Topics Fund of China (Grant RCS2017K008).

J. He, and N. J are with the Department of Systems Design Engineering, University of Waterloo, Waterloo, Canada.

C. Jiang is with the School of Transportation and Logistics, Southwest Jiaotong University, China.

X. Sheng and X. Zhu are with State Key Lab of Mechanical System and Vibration, Shanghai Jiao Tong University, Shanghai, China.

*To whom correspondence should be addressed. E-mail: ning.jiang@uwaterloo.ca, and jiangchaozhe@swjtu.cn communication of the deaf and mute with sign language interpretation [3], and the most common scenario, providing the amputees intuitive and effortless control of the advanced upper-limb prosthesis [4] [5]. Meanwhile, the applications in a boarder range of industry sectors are also being explored [6] [7], with successful commerical products available for non-medical applications [8] [9].

Surface electromyogram (sEMG) signal is the electrical manifestation of functioning muscle contractions, detected noninvasively over the skin surface [5]. As rich gesture-related neural information contained within, sEMG signals are usually used as a control source for AGR, termed as myoelectric control. In the traditional setting, the electrodes for signal collection are attached around the forearm, on the top of the muscle bellies. Recently, comparable classification results were obtained with the electrodes attached on the wrist, expanding the application scenario of myoelectric control [10].

In most myoelectric control studies, pattern recognition (PR) framework were employed from the assumption that the signal pattern of one gesture would be repeatable with the given settings, and distinctive from that of other gestures. Promising results were obtained from PR-based myoelectric control with advanced algorithms in feature extraction [11][12], classification [13][14], and effective experiment setups (optimal electrode configuration [15], brain stimulation [16] and user training [17]). Among these factors, physical channel number was a crucial one for the performance of PR-based myoelectric control scheme. In general, the recognition performance increased with the increasing number of channels, for the collection of addition information about more muscle contractions with enlarged detection area [15]. The common number of channels in PR-based myoelectric control was from four to eight, achieving the offline classification accuracy around 95\% [18]. Recently, promising results with respect to system robustness were obtained from the use of high density (HD) grids (around hundreds of monopolar electrodes) [12][19].

However, for myoelectric control, few studies addressed the scheme with a small number of sEMG channels, such as two channels employed in the most commercial powered prostheses [5]. From the perspective of the practical application, the number of channels was required as few as possible. Decreasing the number of physical channels would reduce the complexity of the system, making it easy to deploy, as well as reduce the power consumption and extend the operation time of 
the system. These features were favorable for many industrial applications, especially the mobile and wearable devices, indicating a big potential market for myoelectric control. Though decreasing the number of channels benefited the practical application, from the perspective of control performance, the small number of channels would reduce the amount of muscular information that could be extracted, leading to the deterioration of the classification accuracy. As such, how to extract the effective information from the limited sources and maintain the performance was the key to the PR-based myoelectric control system with a small number of channels.

It was reported that spatial information of myoelectric signals was important for gesture recognition [15][20]. In this study, we focused on the case of two physical channels, and proposed to enhance its spatial information from two aspects, optimizing the electrode position and applying a spatial filter. Previous studies indicated that the positions of the electrodes was not a critical factor for PR-based myoelectric control when a sufficient number of electrodes was available (usually more than four electrodes) [21]. However, in the case of two channels, the results would be different because of the limited spatial coverage, in which case the optimization of electrode position might be essential for effective myoelectric control. On the other hand, various spatial filters were developed to improve myoelectric control accuracy and robustness by separating the information with respect to different gestures [19][20]. However, most of them required a large number of channels. This study proposed to use a simple spatial filter specifically for a small number of channels, i.e. creating two virtual channels (VC) from two physical channels. We intended to demonstrate that, through the combination of the optimal electrode position and VC technique, PR-based myoelectric control performance with two channels can be enhanced. In addition, the correlation between online and offline performance metrics was investigated in PR-based control scheme. The outcome of this study would be beneficial for the practical use of myoelectric control interface, as well as its algorithm development.

\section{METHOD}

\section{A. Subjects}

Eight able-bodied subjects (seven males and one female, aged from 20 to 30 years old) participated in the study. Before the experiment, the informed consent was obtained from all the

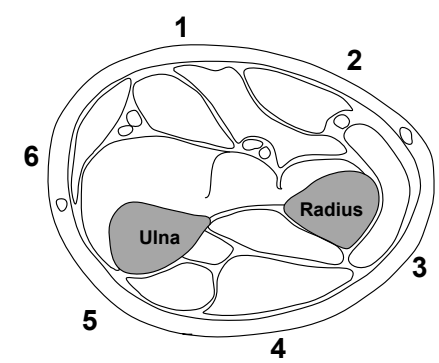

Fig. 1. Cross-sectional view of the placement of six sEMG electrodes. The number represents the position where the electrode is placed.

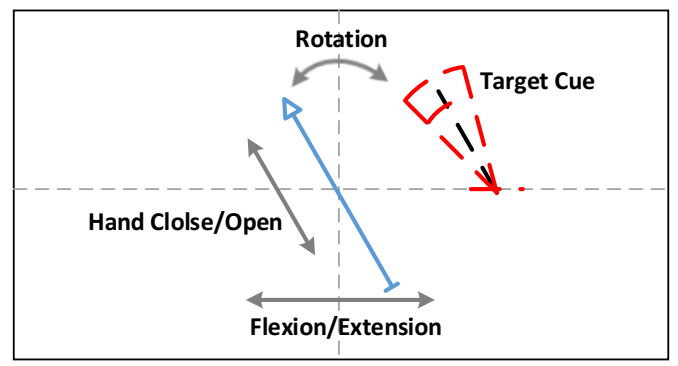

Fig. 2. Interface of online test system. Left-right movements of the arrow is associated to wrist flexion and extension. Rotational movement of the arrow is associated to supination and pronation. Change of the arrow length is associated to hand close and open. A representative target is displayed, and the dash line represents the range of the tolerance.

subjects. The experiment procedures were in accordance with the Declaration of Helsinki.

\section{B. Offline Experiment Protocol}

The subject seated naturally, extending the arm toward the ground. Six surface monopolar electrodes were placed around the forearm, roughly equidistant from each other and approximately $4 \mathrm{~cm}$ to the elbow crease (Fig. 1). Before attaching the electrodes, the skin area was cleaned with alcohol and conductive gel to minimize the electrode-skin impedance and increase the signal quality. Data from six hand and wrist gestures, in addition to the resting class, were collected in this study: hand close, hand open, wrist flexion, wrist extension, pronation, and supination. During the data collection, the subjects were asked to follow the gesture displayed in a monitor in front of them. The contraction of each gesture was held for 5 $\mathrm{s}$ (a trial), followed by a 5-s resting period, after which the next contraction (trial) was performed. In one run, each gesture was performed once and its order was randomized. There was a $30-\mathrm{s}$ rest between two runs to avoid fatigue. For each subject, five runs of data were collected by a commercial bio-signal amplifier (g.USBamp, g.Tec Medical Engineering, Austria) with the signal band-pass filtered at 20 to $450 \mathrm{~Hz}$ and sampled at $1200 \mathrm{~Hz}$.

\section{Online Experiment Protocol}

The online experiment, a control task adapted from [22], followed immediately after the subject finished the offline data collection. It was designed to mimic the practical control scenario for the evaluation of the proposed method. In the experiment, the subject was introduced to a virtual environment (Fig. 2), where the movement of a virtual arrow was associated to the hand and wrist gestures of the subject. The left/right movement of the arrow represented wrist flexion/extension; the clockwise/counter-clockwise movement of the arrow represented wrist supination/pronation. The increase/decrease of the arrow length represented hand open/close. The speed of the arrow was fixed. Before the test, the subject was instructed to be familiar with the system with the classifier calibrated by the offline data of all the six channels. During the test, the subject was asked to move the arrow to the intended target within the tolerance of each direction. Twenty targets were presented to the subject in one session. The targets were the same across different sessions, but the sequence of the targets 


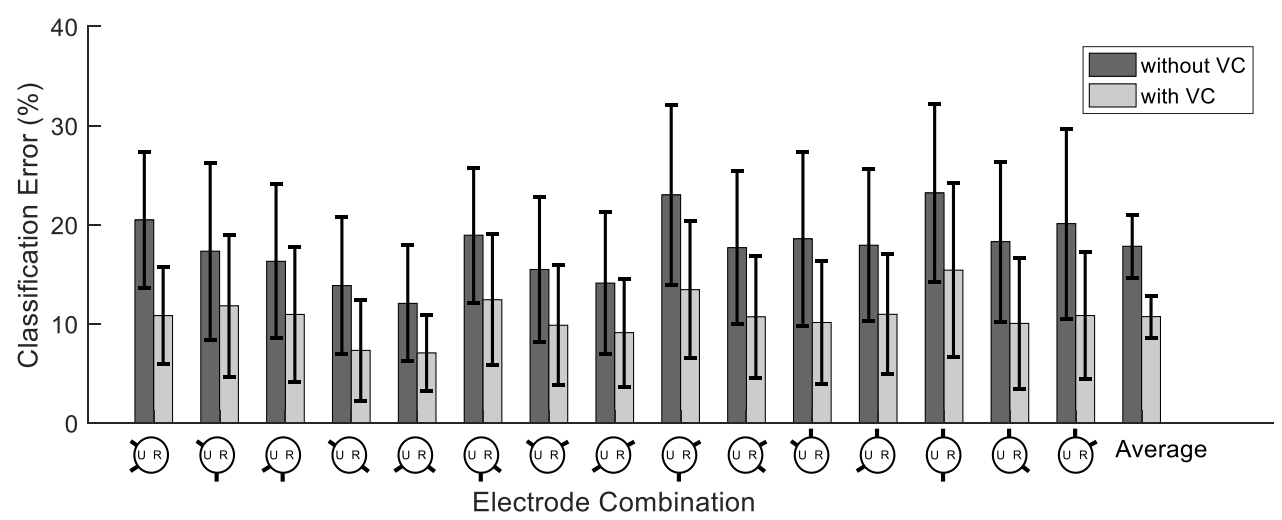

Fig. 3. Error rates of two algorithms (without and with VC) with different electrode positions. The error rates are averaged across subjects. The tick label of horizontal axis displays the positions of two electrodes. The circle represents the cross-sectional view of the arm. The letter $\mathrm{U}$ and $\mathrm{R}$ indicate the position of ulnar and radius, respectively. The black dot represents the positions of the two electrodes used. The last column of each figure represents the error rates averaged across the electrode combination and the subject.

within one session was randomized.

\section{Data Processing}

Acquired EMG signals were segmented into 200-ms analysis windows, with an overlap of $150 \mathrm{~ms}$, resulting in a simulated 50 -ms decision stream. The state-of-the-art time domain (TD) feature sets [23], consisting of mean absolute value (MAV), sign slope change (SSC), zero crossing (ZC), and waveform length (WL), were extracted from each window. The extracted feature vectors were fed to the classifier for recognition. Linear discriminant analysis (LDA) was chosen as the classifier for its low computation cost and comparable performance to other complex classifiers [4].

Two approaches were taken to exploit the spatial information: electrode position optimization and the inclusion of VC. For the effect of electrode position, all possible fifteen combinations out of the six electrodes were considered in this study. Meanwhile, two VCs were formed by making either the average of or the difference between two real channels,

$$
\begin{aligned}
& V_{1}(t)=\left(R_{1}(t)+R_{2}(t)\right) / 2 \\
& V_{2}(t)=\left(R_{1}(t)-R_{2}(t)\right) / 2
\end{aligned}
$$

where $R_{1}(t)$ and $R_{2}(t)$ are the two recorded sEMG signals at time $t$, and $V_{1}(t)$ and $V_{2}(t)$ represent the two VCs. Subsequent feature extraction and classification tasks were then performed with these two new channels.

The objective of this study is to investigate if the combination of electrode position optimization and $\mathrm{VC}$ embedment could improve the myoelectric control performance with only two channels. For this purpose, in offline data analysis, we compared the performance of all the fifteen electrode combinations under two conditions: without $\mathrm{VC}$ and with VC, by classification error rate. A five-fold cross-validation was used, where training and testing data were four runs and one run, respectively. Based on the results of offline analysis, three electrode subsets were selected, the subset with the biggest error rate of the group without VC (worst subset, WS), the subset with the lowest error rate of the group without $\mathrm{VC}$ (optimal subset, OS), and the subset with the lowest error rate of the group with VC (optimal VC subset,
OVS). Subsequently, four cases were tested in online experiment to investigate the improvement of online controllability from the combination of optimal electrode and VC: 1) WS without VC in the following algorithm, 2) OVS without $\mathrm{VC}$ in the following algorithm, 3) OS without $\mathrm{VC}$ in the following algorithm, 4) OVS with VC in the following algorithm. The online performance was assessed by the trajectories of the tip of the arrow and the duration of the arrow movement. Four indices were used to quantify the performance: completion rate, completion time, overshoot, and efficiency coefficient (Table I). A target was considered completed when the subject successfully moved and kept the arrow within the intended tolerance within $20 \mathrm{~s}$. The tolerance range in each direction was corresponded to three analysis window steps, which is $150 \mathrm{~ms}$.

\section{E. Statistical Analysis}

To test the effectiveness of the proposed method, for offline performance, a two-way repeated-measure analysis of variance (ANOVA) was conducted on the error rates. The two factors were positions of the two electrodes (fifteen combinations) and $\mathrm{VC}$ (with or without VC). The main purpose of this ANOVA was to investigate if the inclusion of $\mathrm{VC}$ could improve the classification performance with two electrodes, as it was expected that the positions of the electrodes would affect classification performance. Further, a one-way repeated-measure ANOVA was conducted on error rates to

TABLE I

OnLine Performance Metrics

\begin{tabular}{ll}
\hline \hline Metric & \multicolumn{1}{c}{ Description } \\
\hline $\begin{array}{l}\text { Completion } \\
\text { Rate }\end{array}$ & $\begin{array}{l}\text { the number of completed tasks over the total number } \\
\text { of attempted tasks } \\
\text { Completion } \\
\text { Time }\end{array}$ \\
$\begin{array}{l}\text { the time it took the subject to complete the } \\
\text { successful attempt } \\
\text { the number of occurrences that the tip of the arrow } \\
\text { passed through the target before the dwelling time } \\
\text { was reached } \\
\text { the ratio between the length of the optimal path from } \\
\text { the initial point to the target and the actual trajectory } \\
\text { realized (a value of 100\% indicates a perfect } \\
\text { execution) }\end{array}$ \\
$\begin{array}{l}\text { Efficiency } \\
\text { Coefficient }\end{array}$ \\
\hline \hline
\end{tabular}




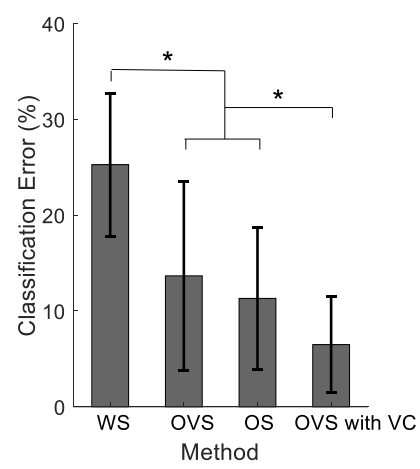

Fig. 4. Comparison of error rates of four methods. The tick label of horizontal axis, WS, OVS, OS, and OVS with VC, represents the classification method using TD feature and LDA classifier with electrode subset WS without VC, with electrode subset OVS without VC, with electrode subset OS without VC, and with electrode subset OVS with VC. The results are averaged across subjects. Star denotes the significant difference between two compared methods $(p<0.05)$.

compare the performances of four methods mentioned Section II (D). A paired t-test was conducted to compare the performance of the proposed method, OVS with VC, with that using four electrodes (averaged across the combinations). For the online performance, a two-way repeated-measure ANOVA was conducted on efficiency coefficient to investigate if there was significant difference between three methods. The method with WS without VC was excluded because the number of successful targets in this case was too poor: on average only one-third of the other three. The two factors were targets ( 20 levels for 20 different targets in one session) and methods. The other three metrics, completion rate, completion time and overshoot, were not considered for their skewed distributions caused by the limit they had. In all the ANOVA models, subject was regarded as a random factor. If significant interaction between two factors was detected, the focused ANOVA would be performed with the fixed level of one factor. If significant difference was detected for the main factor, Turkey comparison was performed. The significance level was set to 0.05 for all the tests.

\section{RESULTS}

\section{A. Offline Performance}

The offline classification error rates varied among different electrode combinations (Fig. 3). The performance of the algorithm with $\mathrm{VC}$ was better than that without $\mathrm{VC}$ on all the electrode combinations. The error rate averaged across the electrode combinations was $17.51 \pm 2.81 \%$ without $\mathrm{VC}$, and $10.15 \pm 1.60 \%$ with VC. The statistical analysis showed that there was no significant interaction between the factors of electrode position and VC. The inclusion of VC significantly decreased the classification errors $(p<0.001)$ regardless of the electrode position.

The selected electrode subsets, WS, OVS and OS, were identified from each subject. The results of the four methods, generated from these subsets (as explained in Section II (D)), were presented in Fig. 4. The combination of optimal electrode

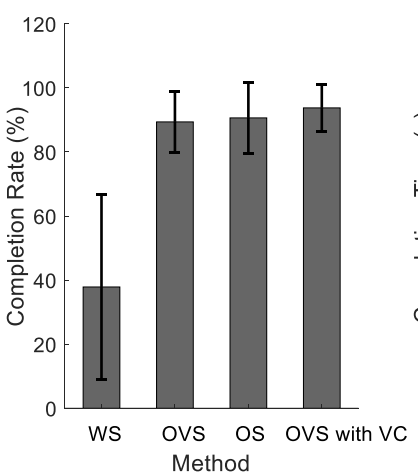

(a)

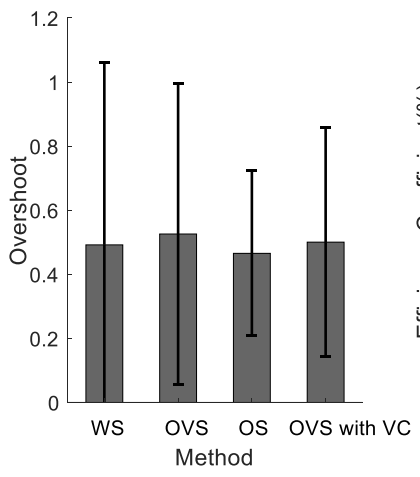

(c)

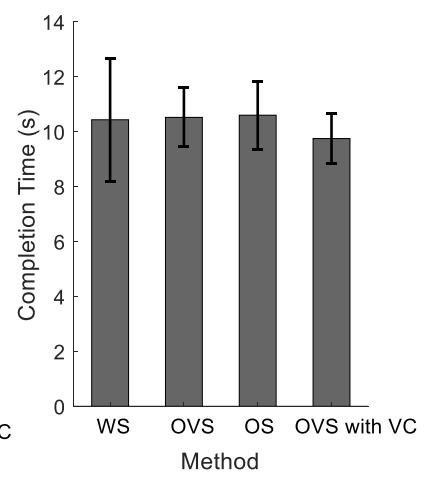

(b)

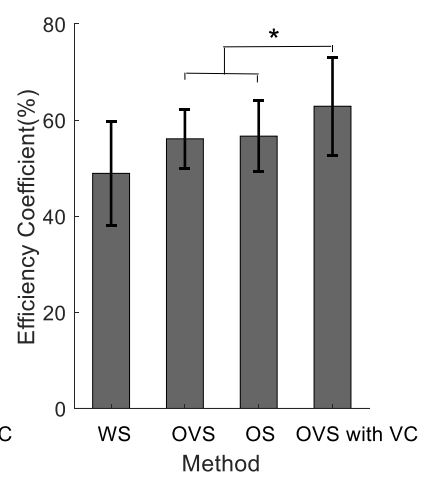

(d)
Fig. 5. Summary of online performance metrics, completion rate (a), completion time (b), overshoot (c), and efficiency coefficient (d). The tick label of horizontal axis, WS, OVS, OS, and OVS with VC, represents the classification method using TD feature and LDA classifier with electrode subset WS without VC, with electrode subset OVS without VC, with electrode subset OS without VC, and with electrode subset OVS with VC. The results are averaged across subjects. Star denotes the significant difference between two compared methods $(p<0.05)$. The method, WS without $\mathrm{VC}$, is excluded in the statistical analysis for the insufficient number of successfully targets.

position and $\mathrm{VC}$ embedment, OVS with $\mathrm{VC}$, obtained the lowest error rate $(6.69 \pm 5.58 \%)$, while the unoptimized electrode placement without VC, WS without VC, obtained the highest error rate $(24.71 \pm 7.45 \%)$. The error rates between the other two methods, OVS without VC and OS without VC, were close. It was indicated in the subsequent statistical analysis that there was significant difference among the methods $(p<0.001)$. Post-hoc comparison showed that the error rate of the proposed method, OVS with VC, was significantly lower than the error rates of the other three, while the error rates of the two methods, OVS without VC and OS without VC, were significantly lower than that of WS without VC. There were no significant difference between OVS without VC and OS without VC.

In addition, to compare the proposed method with the traditional myoelectric control algorithm, the classification error averaged across the combination of four out of six electrodes (fifteen combinations) was calculated, $6.49 \pm 3.9 \%$. The statistical analysis indicated that there was no significant difference between the performance of four electrodes and the proposed method, OVS with VC. 


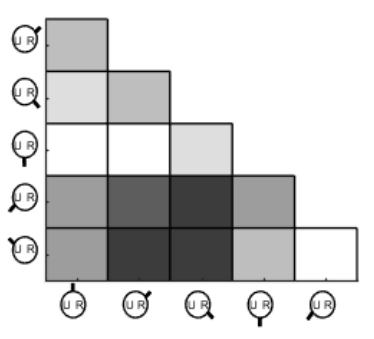

(a)

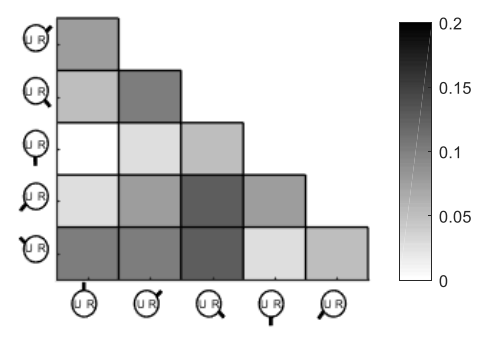

(b)
Fig. 6. Occurrence of the electrode combination in top five lowest error rates for the method using TD feature and LDA classifier with electrode subset OS without VC (a), and with electrode subset OVS with VC (b). The circle at the tickle label represents the cross-sectional view of the arm. The letter $U$ and $R$ represents the position of ulnar and radius. The black dot represents the electrode position.

\section{B. Online Performance}

For all the subjects, the proposed method, OVS with VC, performed better than the other three methods in three online performance metrics (Fig. 5), completion rate, completion time and efficiency coefficient. For the four evaluated methods, WS without VC, OVS without VC, OS without VC, OVS with VC, their completion rate was $37.86 \pm 28.99 \%, 89.38 \pm 9.43 \%$, $90.63 \pm 11.16 \%$, and $93.75 \pm 7.44 \%$, respectively. Similarly, the completion time was $10.43 \pm 2.24 \mathrm{~s}, 10.52 \pm 1.07 \mathrm{~s}, 10.60 \pm$ $1.24 \mathrm{~s}$ and $9.75 \pm 0.91 \mathrm{~s}$, and efficiency coefficient was $46.67 \pm$ $11.90 \%, 55.16 \pm 6.37 \%, 55.65 \pm 7.50 \%$ and $61.63 .95 \pm$ $10.25 \%$. As for the fourth metric, overshoot, its trend was different from the other three. OVS with VC $(0.50 \pm 0.36)$ was inferior to OS without VC $(0.47 \pm 0.26)$, comparable to WS without VC $(0.49 \pm 0.57)$, and superior to OVS without VC $(0.53 \pm 0.47)$. The statistical analysis for efficiency coefficient indicated that there was no significant correlation between the two factors, targets and methods. Post-hoc comparison found that the value of OVS with VC was significantly higher than the values of the other two methods.

\section{Optimal Electrode Combination}

In order to compare the optimal electrode position between the methods with and without $\mathrm{VC}$ in discriminating the six gestures of interest, the top five performances with the lowest classification error rates were selected from the two methods, OS without VC and OVS with VC, respectively. The frequencies of all the channel combinations were counted and presented in Fig. 6. It was indicated that the optimal electrode

TABLE II

SumMary OF CORRELATION ANALYSIS BETWEen ERROR RATE AND Four Online Performance Metrics

\begin{tabular}{|l|c|c|c|c|c|c|c|c|}
\hline \multirow{2}{*}{} & \multicolumn{2}{|c|}{$\begin{array}{c}\text { Completion } \\
\text { Rate }\end{array}$} & \multicolumn{2}{c|}{$\begin{array}{c}\text { Completion } \\
\text { Time }\end{array}$} & \multicolumn{2}{c|}{ Overshoot } & \multicolumn{2}{c|}{$\begin{array}{c}\text { Path } \\
\text { Efficiency }\end{array}$} \\
\cline { 2 - 10 } & $r$ & $p$ & $r$ & $p$ & $r$ & $p$ & $r$ & $p$ \\
\hline$W S$ & 0.67 & 0.09 & 0.17 & 0.71 & 0.28 & 0.55 & 0.10 & 0.84 \\
\hline OVS & 0.72 & 0.04 & 0.21 & 0.63 & 0.01 & 0.97 & 0.11 & 0.79 \\
\hline OS & 0.94 & 0.00 & 0.58 & 0.13 & 0.04 & 0.93 & 0.18 & 0.66 \\
\hline $\begin{array}{l}\text { OVS with } \\
\text { VC }\end{array}$ & 0.44 & 0.28 & 0.17 & 0.70 & 0.05 & 0.90 & 0.05 & 0.92 \\
\hline
\end{tabular}

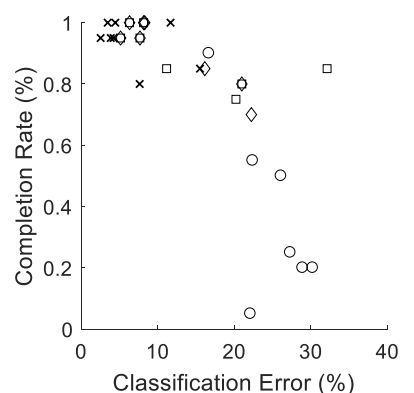

(a)

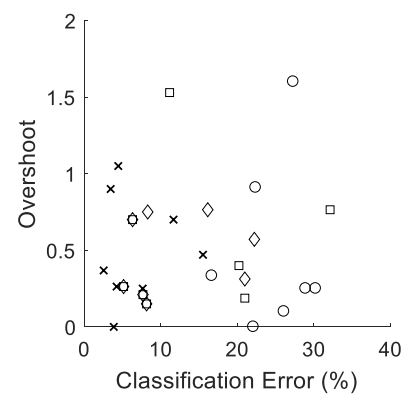

(c)

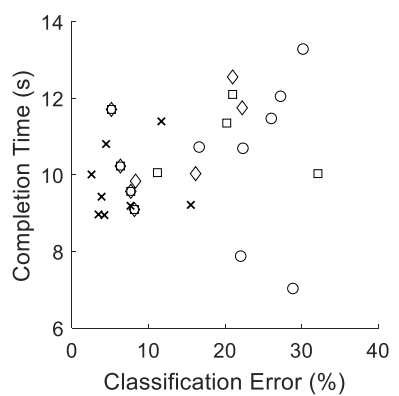

(b)

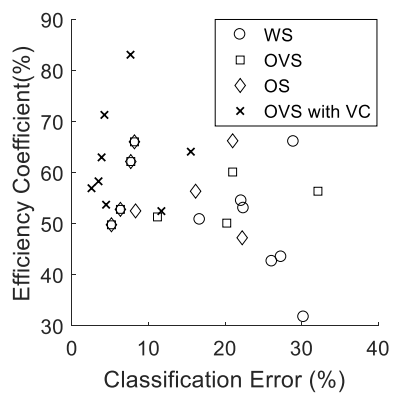

(d)
Fig. 7. Correlation between offline performance metrics, classification error, and the 4 online performance metrics, completion rate (a), completion time (b), overshoot (c), and efficiency coefficient (d). Each data point corresponds to the result from one subject. The classification error is the value averaged over the cross validation, and the value of online performance metrics is averaged over all successful trials performed by the subject using that method.

combinations were similar between two methods. The two electrodes were mostly distant from each other, and one of the two was close to the ulnar bone. The electrode combination, one located in the third column from left to right, and the other locating in the fifth row from upper to down, performed well in both scenarios.

\section{Correlation between Online and Offline Metrics}

Pearson correlation coefficient $(r)$ was performed between error rates (offline) of each method from each subject and the corresponding four online performance metrics. The results are presented in the scatter plots in Fig. 7, and summarized in Table II. The linear correlation was relatively strong for completion rate, and weak for the other three metrics. The highest $r$-value was 0.94 on completion rate for the method OS without VC. The significance was only detected on completion rate for the method OVS without VC and OS without VC. The $p$-value of completion rate was smaller than that of the other three metrics with the same method.

\section{DISCUSSION}

\section{A. Effectiveness of Spatial Information}

In this study, spatial information contained in a two-channel sEMG was exploited through two simple yet effective approaches: optimizing the electrode position and embedding two VCs. The optimization of electrode position was not essential when a sufficiently large number of electrodes were provided. The signal collected by one surface electrode did not 
only include the muscle activities underlying the electrode site, but also include those from the surrounding areas [21], which was referred as cross talk. This characteristic enlarged the detection area of the surface electrode, i.e. the activity of the targeted muscle could be detected by the electrode in its vicinity. With the help of cross talk, when a large number of electrodes were adopted, the total detection area of the system would be large enough to collect a great amount of information, including the effective information for gesture recognition, regardless of whether the electrodes were positioned on the targeted muscle or not. When the number of electrodes decreased, the total detection area of the system was reduced, as well as the amount of information detected. As such, to maintain the recognition performance, the limited electrodes should be optimally placed to collect the most effective information. The necessity of electrode position optimization with a small number of electrodes was confirmed by the performance difference between the scenarios of OS and WS. No matter online or offline, the performance of the method with the electrode subset OS was significantly better than the performance of the method with the electrode subset WS. As indicated in Fig. 6, the most common electrode combination was the pair located on the two sides of the forearm, opposite to each other. One was around the extensor carpi radialis, and another was around the flexor carpi ulnaris and flexor digitorum communis.

The simple VC algorithm was proposed to further exploit spatial information to improve myoelectric control performance. The embedment of $\mathrm{VC}$ in myoelectric signals could be regarded as the product of raw signals and the matrix,

$$
\left\lceil\begin{array}{cc}
1 & 0 \\
0 & 1 \\
0.5 & 0.5 \\
0.5 & -0.5
\end{array}\right]
$$

such format was used in some other myoelectric studies, where the matrix could be obtained with the optimization of training data [19], or dimension reduction algorithms [24], and operated on features. High accuracy and robustness were achieved with these algorithms in myoelectric control. However, most of these algorithms were not designed for the limited channel case, where the dimension of the original data was low. In this study, a fixed matrix was used to extend the dimension of output. When the subsequent feature extracted was linear, its effect might be limited for the features from the VC could be obtained by linear operations of the features from the physical channels. When the feature extracted was nonlinear, the features from VC contained information from both physical channels and could not be replicated from the features of these channels. The additional information extracted from VC provided the performance improvement of PR-based myoelectric control. As the features in TD were all nonlinear, the effect of the algorithm was significant.

When considering the top five optimal subsets, the electrode combinations for two methods, OS without VC and OVS with $\mathrm{VC}$, were similar. This similarity indicated that the inclusion of VC didn't change the sets of original optimal electrode positions. Therefore, the benefits of these two methods: optimal electrode position and VC, could be combined (OVS with VC), achieving classification performance similar to the traditional configuration of four electrodes $(\mathrm{p}<0.05)$. This indicated that the number of channels was not conclusive for the recognition performance. In fact, the relationship between the number of electrodes and recognition performance was a complicated topic for myoelectric control, addressing the factors such as the electrode position [15], size and orientation [25]. The effect of the number of channels on the proposed methods will be investigated in future studies.

As mentioned above, the aim of the electrode optimization and $\mathrm{VC}$ embedment was to collect and extract more effective information with limited resources. As mentioned above, the aim of the electrode optimization and $\mathrm{VC}$ embedment was to collect and extract more effective information with limited resources. If a larger number of channels was adopted, the amount of information it captured would be sufficient to decipher the gestures, and the classification performance would be already high, such that the effect of the proposed method might not as effective as that on the two-channel system. The six hand and wrist gestures were selected for their high recognition accuracy [18], making them more likely to be adopted in practical application. However, the proposed method was not developed based on one specific gesture. As such, it was also applicable to the recognition of other gestures, such as those involving finger motions. However, further investigation would be required to fully understand if the proposed methods were beneficial in recognizing finger motions.

Robustness has been identified as one of the key limitations of current state-of-the-art in myoelectric control [26]. There were many factors against which myoelectric control algorithms need to be robust, such as muscle fatigue, electrode shift, and arm position change. All these factors would change the characteristics of the input signal, resulting inconsistency between training data and testing data, subsequently degradation in system performance. Many methods were proposed to make the system robust against these factors, and most of them focused on the selection of feature sets [12][27] and classifier [13][14]. As the proposed methods are part of the system acquisition setting and data preprocessing, upstreaming to features and classifier, it is quite possible that they can be combined with the effective features and classifiers to further improve the system robustness and overall system performance.

\section{B. Relationship between online and offline metrics}

Relationship between online and offline metrics was still an open question for myoelectric control studies. Previous studies reported that the good performance in offline metrics was not necessarily mapped to the good performance in online metrics with simultaneous and proportional myoelectric control scheme [22], where instead of the discrete value, the single gesture, the continuous value, the angle or the torque of the finger and wrist joint, was predicted. The difference between offline and online test was the feedback provided to the subject in the online scenario. In offline scenario, there was no feedback provided to 
the subject. While in online scenario, the subject would receive real-time feedback and adjust their movements according to the feedback to achieve the desired outcome. This was not possible in the offline scenario. In addition, through the interaction with the system, the subject could be adapted to the control scheme in online scenario. As such, the online scenario was more complex than the offline scenario.

In this study, high linear correlation was observed between offline classification error rate and online completion rate. Further, for the methods OS without VC and OVS without VC, the statistical significance was detected. However, the correlations between the error rate and the other three metrics were weak and insignificant. We speculated that this was caused by the insufficient feedback provided by the PR-based myoelectric control scheme. Compared to the simultaneous and proportional control scheme, the feedback of PR-based control scheme was discrete, not continuous, which limited the adaptability of the subject [28]. As such, the effect of feedback was mitigated, and the completion rate was correlated to the classification error rate. This indicated that the correlation between offline and online performance for PR-based myoelectric control scheme was stronger than that for simultaneous and proportional myoelectric control scheme. On the other hand, as only successful trials were accounted, of which most go smoothly without long time in modulating muscle contractions, the correlation between completion time and error rate was weak, as well as between efficiency coefficient and error rate. For the overshoot, it was correlated with the transition between the active gesture and the rest state, which was not fully reflected on error rate. In addition, for the method WS without VC and OVS with VC, the values of the coefficient on completion rate were relatively low and insignificant. As displayed in Fig. 7(a), most samples of OVS with VC gathered at the left upper corner, where the value of completion rate was close to $100 \%$, while the samples of WS were in the right bottom part of the figure, where the error rate exceeded $20 \%$. It indicated that when the error rates were too low or too high, its change had limited effect on the improvement of the online performance.

The significant correlation between online completion rate and offline classification accuracy within the range of around $10 \%$ to $20 \%$ would simplify the test of the advanced algorithms for myoelectric control. As online test was much closer to the scenario of the real-world application, its results were more important than the results of offline test. However, the cost of offline test was normally much lower than that of online test, and algorithm optimization can be performed much faster in offline. Online testing and optimization is time and resource intensive, consequently costly. The significant correlation between online and offline results found in this study, indicate that the offline performance is transferable to online performance, allowing algorithm optimization to be done offline, which will significantly improve algorithm development efficiency.

\section{CONCLUSION}

This study focused on enhancing the performance of myoelectric control using only two channels, which has potential for mobile and wearable application scenarios, beyond prosthesis control. The experiments indicated that the proposed method, combination of electrode position optimization and VC embedment, significantly improve system performance, and it was comparable to that of the traditional method with four electrodes. In addition, strong linear correlation was found between the offline and online performance metrics, error rate and completion rate. The performance correlation between online and offline would improve the efficiency of the algorithm development.

\section{REFERENCES}

[1] H. Liang, J. Yuan, D. Thalmann, and N. Magnenat-thalmann, “AR in Hand : Egocentric Palm Pose Tracking and Gesture Recognition for Augmented Reality Applications," ACM Multimed. Conf., pp. 6-7, 2015.

[2] R. Song, K. Y. Tong, X. Hu, and W. Zhou, "Myoelectrically controlled wrist robot for stroke rehabilitation," J. Neuroeng. Rehabil., vol. 10, no. 1, 2013.

[3] Y. Li, X. Chen, X. Zhang, K. Wang, and Z. J. Wang, "A sign-component-based framework for Chinese sign language recognition using accelerometer and sEMG data," IEEE Trans. Biomed. Eng., vol. 59, no. 10, pp. 2695-2704, 2012.

[4] M. Hakonen, H. Piitulainen, and A. Visala, "Current state of digital signal processing in myoelectric interfaces and related applications," Biomed. Signal Process. Control, vol. 18, pp. 334-359, 2015.

[5] D. Farina et al., "Man/machine interface based on the discharge timings of spinal motor neurons after targeted muscle reinnervation," Nat. Biomed. Eng., vol. 25, no. February, 2017.

[6] C. Yang, S. Chang, P. Liang, Z. Li, and C. Y. Su, "Teleoperated robot writing using EMG signals," in 2015 IEEE International Conference on Information and Automation, ICIA 2015 - In conjunction with 2015 IEEE International Conference on Automation and Logistics, 2015, pp. 2264-2269.

[7] P. Ben-Tzvi and Z. Ma, "Sensing and Force-Feedback Exoskeleton (SAFE) Robotic Glove," IEEE Trans. Neural Syst. Rehabil. Eng., vol. 23, no. 6, pp. 992-1002, Nov. 2015.

[8] Thalmic Labs Inc., "Myo ${ }^{\mathrm{TM}}$ Gesture Control Armband - Wearable Technology by Thalmic Labs," Thalmic Labs Inc., 2013. [Online]. Available: https://www.myo.com/\%0Awww.myo.com.

[9] "Athos Training System," https://www.liveathos.com/. [Online]. Available: https://www.liveathos.com/.

[10] S. Jiang et al., "Feasibility of Wrist-worn, Real-time Hand and Surface Gesture Recognition via sEMG and IMU Sensing," IEEE Trans. Ind. Informatics, vol. 3203, no. c, pp. 1-1, 2017.

[11] A. Phinyomark, F. Quaine, S. Charbonnier, C. Serviere, F. Tarpin-Bernard, and Y. Laurillau, "EMG feature evaluation for improving myoelectric pattern recognition robustness," Expert Syst. Appl., vol. 40, no. 12, pp. 4832-4840, Sep. 2013.

[12] J. He and X. Zhu, "Combining Improved Gray-Level Co-Occurrence Matrix with High Density Grid for Myoelectric Control Robustness to Electrode Shift," IEEE Trans. Neural Syst. Rehabil. Eng., vol. 11, no. 4, pp. 1-1, 2016.

[13] J. L. Betthauser et al., "Limb Position Tolerant Pattern Recognition for Myoelectric Prosthesis Control with Adaptive Sparse Representations from Extreme Learning," IEEE Trans. Biomed. Eng., vol. 65, no. 4, pp. 770-778, 2018.

[14] S. Amsuss, P. Gobel, N. Jiang, B. Graimann, L. Paredes, and D. Farina, "Self-Correcting Pattern Recognition System of Surface EMG Signals for Upper Limb Prosthesis Control," IEEE Trans. Biomed. Eng., vol. 9294, no. c, pp. 1-1, 2014.

[15] J. He, X. Sheng, X. Zhu, and N. Jiang, "Electrode density affects the robustness of myoelectric pattern recognition system with and without electrode shift," IEEE Journal of Biomedical and Health Informatics, 2018.

[16] L. Pan, D. Zhang, X. Sheng, and X. Zhu, "Improved Myoelectric Control for Amputees through Transcranial Direct Current Stimulation.," IEEE Trans. Biomed. Eng., vol. 9294, no. c, pp. 1-11, 2015. 
[17] J. He, D. Zhang, N. Jiang, X. Sheng, D. Farina, and X. Zhu, "User adaptation in long-term, open-loop myoelectric training: implications for EMG pattern recognition in prosthesis control," J. Neural Eng., vol. 12, no. 4, p. 46005, Aug. 2015.

[18] G. Li, A. E. Schultz, and T. a Kuiken, "Quantifying pattern recognition-based myoelectric control of multifunctional transradial prostheses.," IEEE Trans. Neural Syst. Rehabil. Eng., vol. 18, no. 2, pp. 185-192, Apr. 2010.

[19] L. Pan, D. Zhang, N. Jiang, X. Sheng, and X. Zhu, "Improving robustness against electrode shift of high density EMG for myoelectric control through common spatial patterns.," J. Neuroeng. Rehabil., vol. 12, no. 1, p. 110, 2015.

[20] A. Stango, F. Negro, and D. Farina, "Spatial Correlation of High Density EMG Signals Provides Features Robust to Electrode Number and Shift in Pattern Recognition for Myocontrol," IEEE Trans. Neural Syst. Rehabil. Eng., vol. 23, no. 2, pp. 189-198, Mar. 2015.

[21] T. R. Farrell and R. F. F. Weir, "A comparison of the effects of electrode implantation and targeting on pattern classification accuracy for prosthesis control.," IEEE Trans. Biomed. Eng., vol. 55, no. 9, pp. 2198-211, Sep. 2008.

[22] N. Jiang, I. Vujaklija, H. Rehbaum, B. Graimann, and D. Farina, "Is accurate mapping of EMG signals on kinematics needed for precise online myoelectric control?," IEEE Trans. Neural Syst. Rehabil. Eng., vol. 22, no. 3, pp. 549-58, May 2014.

[23] B. Hudgins, P. Parker, and R. N. Scott, "A new strategy for multifunction myoelectric control.," IEEE Trans. Biomed. Eng., vol. 40, no. 1, pp. 82-94, Jan. 1993.

[24] D. Zhang, X. Zhao, J. Han, and Y. Zhao, "A comparative study on PCA and LDA based EMG pattern recognition for anthropomorphic robotic hand," in Proceedings - IEEE International Conference on Robotics and Automation, 2014, pp. 4850-4855.

[25] A. J. Young, L. J. Hargrove, and T. a Kuiken, "The effects of electrode size and orientation on the sensitivity of myoelectric pattern recognition systems to electrode shift.," IEEE Trans. Biomed. Eng., vol. 58, no. 9, pp. 2537-44, Sep. 2011.

[26] J. Ning, S. Dosen, K.-R. Muller, and D. Farina, "Myoelectric Control of Artificial Limbs-Is There a Need to Change Focus? [In the Spotlight]," IEEE Signal Process. Mag., vol. 29, no. 5, pp. 152-150, Sep. 2012.

[27] Y. Gu, D. Yang, Q. Huang, W. Yang, and H. Liu, "Robust EMG pattern recognition in the presence of confounding factors: features, classifiers and adaptive learning," Expert Syst. Appl., vol. 96, pp. 208-217, 2018.

[28] J. M. Hahne, M. Markovic, and D. Farina, "User adaptation in Myoelectric Man-Machine Interfaces," Sci. Rep., vol. 7, no. 1, p. 4437, 2017.

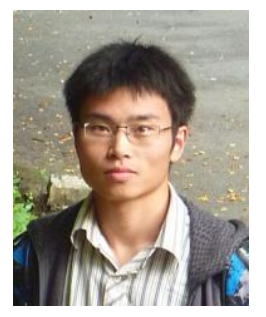

Jiayuan He received the Bachelor's degree in mechanical engineering and automation from Nanjing University of Aeronautics and Astronautics, Nanjing, China, in 2010, and the $\mathrm{Ph} . \mathrm{D}$. degree in mechanical engineering from Shanghai Jiao Tong University, Shanghai, China, in 2016. He is currently a Senior Research Scientist with Engineering Bionics Lab at the University of Waterloo, Canada. His research interests include biomedical signal processing and myoelectric prosthesis control.

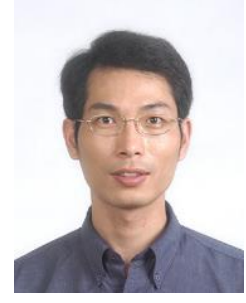

Xinjun Sheng received the B.Sc., M.Sc. and $\mathrm{Ph} . \mathrm{D}$. degrees in mechanical engineering from Shanghai Jiao Tong University, Shanghai, China, in 2000, 2003 and 2014, respectively. In 2012, he was a visiting scientist in Concordia University, Montreal, QC, Canada. He is currently an Associate Professor with the School of Mechanical
Engineering, Shanghai Jiao Tong University. His current research interests include robotics, and biomechatronics. Dr. Sheng is a member of IEEE, RAS, EMBS, and IES.

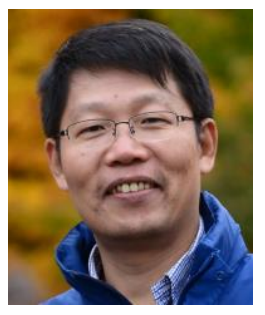

Xiangyang Zhu received the B.S. degree from the Department of Automatic Control Engineering, Nanjing Institute of Technology, Nanjing, China, in 1985, the M.Phil. degree in instrumentation engineering and the Ph.D. degree in automatic control engineering, both from Southeast University, Nanjing, China, in 1989 and 1992, respectively. From 1993 to 1994, he was a postdoctoral research fellow with Huazhong University of Science and Technology, Wuhan, China. He joined the Department of Mechanical Engineering as an associate professor, Southeast University, in 1995. Since June 2002, he has been with the School of Mechanical Engineering, Shanghai Jiao Tong University, Shanghai, China, where he is currently a Changiiang Chair Professor and the director of the Robotics Institute. His current research interests include robotic manipulation planning, human-machine interfacing, and biomechatronics. Dr. Zhu received the National Science Fund for Distinguished Young Scholars in 2005.

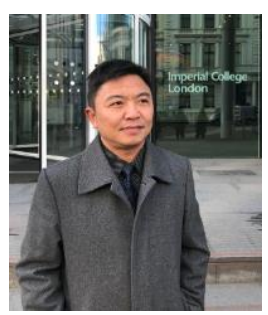

Chaozhe Jiang is an Associate Professor of Transportation at Southwest Jiaotong University, China. Currently he is also a research fellow and executive director of iTSS-RC lab in the department of Civil and Environmental Engineering at University of Waterloo, Canada. He received $\mathrm{PhD}$. Degree in Management Science and Engineering from Southwest Jiaotong University in 2006 and MASc degree in Civil/Transportation Engineering at University of Waterloo. His research interests include sensors, ITS, transportation planning, logistics and SCM finance, systems engineering, big data and AI

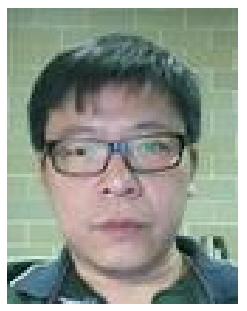

Ning Jiang received his B.S. degree in electrical engineering from Xi'an Jiaotong University, Xi'an, China, in 1998, and his M.Sc. and Ph.D. degrees in engineering from the University of New Brunswick, Fredericton, NB, Canada, in 2004 and 2009, respectively. He was Research Assistant Professor at Aalborg University, Denmark from Apr. 2009 to Aug. 2010, a Marie Curie Fellow Otto Bock Healthcare GmbH, Germany from Sep. 2010 to Oct. 2012, and a research scientist with Department of Neurorehabilitation Engineering, University Medical Center Göttingen, Göttingen, Germany from Nov. 2012 to Apr. 2015. Since May 2015, he is the Director of Engineering Bionics Lab, and an Assistant Professor, University of Waterloo, Waterloo, Canada. His research interests include signal processing of electromyography and electroencephalogram, advanced prosthetic control, neuromuscular modeling and brain computer interfaces for neurorehabilitation. He is currently Associated Editor for IEEE Journal of Biomedical and Health Informatics, the Brain Computer Interface, and Frontiers in Neuroscience. 